\title{
Hauptströmungen \\ im Gespräch zwischen Theologie und Naturwissenschaften im angelsächsischen Sprachraum
}

Zum 100. Geburtstag von Karl Heim

Prof. Dr. Hans S c h w a r z, Lutheran Theological Seminary, Capital University, Columbus, Ohio 43209

Trotz seiner imponierenden Lebensleistung ist Karl Heim ein großer Einzelgänger geblieben. Obwohl von den vorherrschenden Strömungen nicht unbeeinflußt, hat er sich keiner Schule angeschlossen. Eine eigene Schule zu bilden, lag ihm nicht. Zudem waren seine Gedankengänge, die umfassende philosophische und naturwissenschaftliche Vorkenntnisse erforderten, ungeeignet, eine eigene Schulbildung in Gang zu setzen. So wurde er zwar von vielen Studenten verehrt und hat auch ganze Pfarrersgenerationen geprägt, doch ist es um ihn verhältnismäßig schnell ruhig geworden. Erst in den letzten Jahren kann man von so etwas wie einer Wiederentdeckung Karl Heims sprechen ${ }^{1}$. Der Grund hierfür scheint darin zu liegen, daß man die Notwendigkeit des Weltbezugs in der Theologie neu entdeckt hat, die bei der lange vorherrschenden neo-reformatorischen Theologie Barths und Bultmanns trotz ihrer unzweifelhaften Verdienste leider zu kurz gekommen ist. Leute wie Sigurd Daecke, Günter Altner, oder auch der von Heim herkommende Ulrich Mann, scheinen den so sträflich vernachlässigten Dialog mit den Naturwissenschaften wieder tatkräftig voranzutreiben. Dabei ist man sich allerdings oft nicht bewußt, daß dieser Dialog in anderen Ländern überhaupt nicht unterbrochen war. Der folgende Beitrag möchte hier auf den angelsächsischen Sprachraum hinweisen, in dem das Gespräch mit den Naturwissenschaften sich immer großen Interesses erfreute, und dessen Vertreter bej diesem Gespräch in Deutschland leider viel zu wenig bekannt sind. Wir wollen hier keinen lüdkenlosen Literaturbericht bieten, da ein solcher den verfügbaren Raum sprengen würde, sondern nur kurz mit den dort vertretenen Hauptrichtungen vertraut machen.

1 Vgl. Karl Heim, Du Herr bist Kraft und Leben. Sein Leben und Werk, dargestellt und ausgew. v. Gert Schörle (Stuttgart 1961). Karl Heim. Ein Christuszeuge im Spannungsfeld zwischen Glaube und Wissen, hg. v. Reinhard Hildenbrand, Adolf Köberle, Alfred Ringwald, Gert Schörle u.a. (Metzingen 1964). Friedrich Hauss, Karl Heim (Gießen 1960). Hermann Timm, Glaube und Naturwissenschaft in der Theologie Karl Heims. Mit einem Vorwort v. H. E. Tödt (Suttgart 1968). Adolf Köberle, Karl Heim. Denker und Verkündiger aus evangelischem Glauben (Hamburg 1973). 
Wenn dadurch das Gespräch in Deutschland ein wenig profitieren würde, läge das sicher auch im Sinne von Karl Heim.

Obgleich die neo-reformatorische Theologie auch im angelsächsischen Sprachraum beträchtlichen Einfluß hatte, führte das zu keiner Beeinträchtigung des Gesprächs zwischen Theologie und Naturwissenschaften. Jährliche Vorlesungsreihen, zu denen jeweils ein hervorragender Gelehrter eingeladen wird, und die von wohlhabenden Privatleuten an Universitäten errichtet und finanziert werden, sorgen ihrerseits für einen Fortgang des Gesprächs. So wurden 1961 die John Danz Lectures an der Universität von Washington in Sattle errichtet, um jährlich einen Gelehrten von nationalen oder internationalen Ruf dorthin zu bringen, der sich mit den Möglichkeiten beschäftigen soll, von Naturwissenschaften und Philosophie her zu einem rationalen Weltverständnis vorzustoßen ${ }^{2}$. Daß als erster Gelehrter zu dieser Vortragsreihe Julian Huxley eingeladen wurde, zeigt die liberal humanistische Intention dieser Stiftung. Wesentlich älter und konservativer sind die McNair Lectures an der Universität von North Carolina. Sie sollen sich der gegenseitigen Abhängigkeit von Naturwissenschaften und Theologie widmen und Existenz und Attribute Gottes aus der Natur beweisen, soweit dies möglich sei ${ }^{3}$. Die Gifford Lectures in Schottland, die dem Gebiet der natürlichen Theologie gewidmet sein sollen ${ }^{4}$, und die Bampton Lectures in Oxford (England), die allgemein apologetischen Charakter haben, sollten in diesem Zusammenhang wenigstens erwähnt werden.

Oft findet man Theologen oder Naturwissenschaftler, die ein Zweitstudium in der anderen Disziplin abgelegt haben. So ist Ian G. Barbour mit einem Bachelor of Divinity Grad von der Yale Divinity School und mit einem Doctor of Philosophy in Physik von der Universität von Chicago ausgewiesen zur Zeit Vorstand der Abteilung für Religion und Professor für Physik am Carleton College in Northfield, Minnesota. Die Theologen Edward LeRoy Long, Jr., und M. Holmes Hartshorne widmeten sich einem ähnlichen Doppelstudium. Schließlich sei hier noch William G. Pollard erwähnt, der Executive Director am Oak Ridge Institute of Nuclear Studies in Oak Ridge, Tennessee, ist und gleichzeitig als Associate Minister an einer Gemeinde der Episkopalkirche tätig ist.

Auch darf man die zahlreichen Symposia nicht vergessen, zu denen Theologen und Naturwissenschaftler zusammenkommen, deren Beiträge

? Vgl. Julian Huxley, The Human Crisis, 1.

${ }^{3}$ C. A. Coulson, Science and Christian Belief, Vorwort.

4 Für die Weite der Vortragsreihe sei hier nur anmerkungsweise angedeutet, daß neben Charles E. Raven, Natural Religion and Christian Theology (Gifford Lectures 1951), auch Rudolf Bultmann, Geschichte und Eschatologie (Gifford Lecutres 1955), als Vortragende eingeladen werden konnten. 
dann oft als Sammelbände veröffentlicht werden. Hier ist etwa der von Dale White herausgegebene Band Dialogue in Medicine and Theology zu nennen, der die Vorträge enthält, die anläßlich eines von der Methodistenkirche veranstalteten Symposiums gehalten wurden, das vom 5.7. April 1967 in der Mayo Klinik und im Rochester Methodist Hospital in Rochester, Minnesota, stattfand. Von größerer Reichweite sind die drei Bände Evolution after Darwin, die von dem Anthropologen Sol Tax herausgegeben wurden und die Vorträge und Diskussionen der University of Chicago Centennial beinhalten, die anläßlich des hundertsten Jahrestages der Veröffentlichung von Darwins The Origin of Species abgehalten wurden. Hervorragende Wissenschaftler aus allen Teilen der Welt trugen zum Gelingen dieser Veranstaltung bei. Im dritten Band dieser Dokumentation werden auch philosophische und theologische Implikationen der Entwicklungslehre bedacht, so etwa in Julian Huxleys Beitrag "The Evolutionary Vision" (Bd. III, 249-262). Selbst Verlage fördern solche Symposia, wie etwa der von Ian G. Barbour herausgegebene Sammelband Science and Religion; New Perspectives on a Dialogue zeigt. Die darin vertretenen Theologen, Philosophen und Naturwissenschaftler repräsentieren eine weite Spanne von Glaubensrichtungen, von dem dem orthodoxen Glauben angehörigen Genetiker Theodosius Dobzhansky bis zu dem liberalen Protestanten Harold K. Schilling, und suchen eine Grundlage für die Diskussion der brennenden Fragen im Gespräch zwischen Theologie und Naturwissenschaften zu schaffen. In diesem Zusammenhang muß auch die von Harlow Shapeley herausgegebene Aufsatzsammlung Science Ponders Religion genannt werden, die 18 Aufsätze führender amerikanischer Naturwissenschaftler enthält, die sich mit Laien und freisinnigen Theologen im Institute of Religion in an Age of Science auf Star Island vor der Küste von New Hampshire jeden Sommer zu Seminaren treffen, um eine Zusammenarbeit zwischen allen Glaubensrichtungen und Vereinigungen voranzutreiben ${ }^{5}$.

Wesentlich konservativer sind die Aufsätze, die in den beiden Sammelbänden Evolution and Christian Thought Today (hg. v. Russell L. Mixter) und The Encounter Between Christianity and Science (hg. v. Richard $\mathrm{H}$. Bube) enthalten sind. Herausgeber und Mitarbeiter dieser Bände weisen einen strikten Fundamentalismus zurüdk und wollen zeigen, daß ihr christlicher Glaube und ihre naturwissenschaftlichen Erkenntnisse sich gegenseitig nicht ausschließen, sondern eher in einem komplementären Verhältnis zueinander stehen. Obwohl etwa zögernd die Möglichkeit einer Entstehung des Lebens aus unbelebter Materie zugegeben wird, lehnt man doch meist eine strikte Evolution des Menschen von den Prähominiden ab und ersetzt evolutionäres Denken durch

s Vgl. »Some Roots of Zygon«, in Zygon, Bd. I, 1966, $117 \mathrm{ff}$. 
*scientific creationism $^{6}$. Der Zweck der beiden Aufsatzbände ist, der Welt zu zeigen, daß prominente Naturwissenschaftler auch gute Nachfolger Christi sein können und in seiner Kirche tatkräftig mitarbeiten? Die Beiträge stammen meist von angesehenen Männern aus allen Bereichen der Naturwissenschaften, die entweder der American Scientific Affiliation angehören oder ihr nahestehen. Die American Scientific Affiliation ist eine Vereinigung, die sich zum Ziel setzt: 1. Die Bestrebungen vieler zusammenzufassen und zu organisieren, die sich bemühen, die Fakten der Naturwissenschaften und der Heiligen Schrift aufeinander zu beziehen. 2. Das Studium des Verhältnisses zwischen naturwissenschaftlichen Fakten und der Heiligen Schrift zu fördern und zu ermutigen. 3. Die Verbreitung der Ergebnisse solcher Studien voranzutreiben ${ }^{8}$. Associate Member der Vereinigung kann jeder werden, der ein aktives Interesse an den Absichten der Vereinigung zeigt. Als reguläres Mitglied muß man ein abgeschlossenes Hochschulstudium in den Natur- oder Sozialwissenschaften nachweisen können und gegenwärtig mit wissenschaftlicher Arbeit beschäftigt sein. Um ein Fellow der Vereinigung werden zu können, muß man in den Natur- oder Sozialwissenschaften promoviert haben, gegenwärtig mit wissenschaftlicher Arbeit beschäftigt sein und durch die Mitglieder zum Fellow gewählt werden. Als Mitglied bestätigt man folgendes Glaubenszeugnis: Die Heilige Schrift ist das inspirierte Wort, die einzig unfehlbare Glaubens- und Verhaltensregel. Jesus Christus ist der Sohn Gottes und durch seine Versöhnung ist er der eine und einzige Mittler zwischen Gott und Mensch ${ }^{9}$. Daß es sich bei dieser Vereinigung um keine unbeträchtliche Gruppe von angesehen Naturwissenschaftlern in den USA handelt, zeigt sich auch darin, daß ihr offizielles Organ, das Journal of the American Scientific Affiliation, eine bezahlte Subskriptionsrate von 2700 Exemplaren hat.

Von ganz anderer theologischer Ausrichtung ist das Center for Advanced Studies in Theology and the Sciences an der unitarischen Meadville Theological School of Lombard College in Chicago, Illinois. Ziel dieses Instituts ist die Wiederbelebung einer religiösen Synthese. Dabei sollen die negativen und positiven Eintlüsse von Technologie und Kybernetik auf den Menschen und auf seine heutigen Wertsysteme ständig neu bedacht werden. Das Zentrum arbeitet eng mit der Universität

- Russell L. Mixter, Evolution and Christian Theology, 189.

7 Richard H. Bube, The Encounter Between Christianity and Science, 11: $*$ It is the purpose of the authors to witness to the world that dedicated men of science can also be dedicated disciples of Jesus Christ and members of His church *.

8 Artikel der Konstitution der American Scientific Affiliation nach Edward LeRoy Long, Jr., Beliefs of American Scientists, $107 \mathrm{ff}$.

- The Holy Scriptures are the inspired Word of God, the only unerring guide of faith and conduct. Jesus Christ is the Son of God and through His Atonement is he the one and only Mediator between God and Man*. 
von Chicago zusammen, obwohl gegenwärtig eine Verlegung an die amerikanische Ostküste erwogen wird. Der Direktor des Zentrums, Ralph Wendell Burhoe ist zugleich Herausgeber von Zygon. Journal of Religion and Science, das bei der University of Chicago Press erscheint. Im weiteren Herausgeberkreis dieser Zeitschrift tauchen Namen von Naturwissenschaftlern und Theologen auf, die auch am Institute of Religion in an Age of Science auf Star Island mitarbeiten. Obwohl die Zeitschrift und das Zentrum neueren Datums sind, wird dort von hervorragenden Naturwissenschaftlern, Philosophen und Theologen erstklassige Arbeit geliefert, die oft einen liberal-unitarischen Eindruck hinterläßt ${ }^{10}$.

Wenn wir nun mit aller Vorsicht gewisse Hauptströmungen unter den verschiedenen Theologen, Naturwissenschaftlern und Philosophen in ihrem Bemühen um ein Gespräch zwischen Theologie und Naturwissenschaften aufzeigen, so kann das hier nur in sehr selektiver und damit subjektiver Weise geschehen. Auch das zur Ergänzung beigefügte Literaturverzeichnis ist in dieser Hinsicht nicht vollständig, da ein Erfassen der gesamten in den letzten zwanzig oder fünfundzwanzig Jahren über dieses Thema geschriebenen Bücher das Verzeichnis mindestens verdoppeln würde. Doch scheinen sich im Gespräch drei Hauptlinien zu ergeben: Entweder man faßt die Inhalte von Theologie und Naturwissenschaften als Kontraste auf, oder man sieht in beiden parallellaufende Erscheinungen, oder man versucht, den Inhalt der Theologie auf naturwissenschaftlicher Basis zu gründen. Daneben gibt es noch die Extremfälle des Fundamentalismus und des Atheismus, mit denen wir uns hier nicht befassen wollen.

\section{I \\ Naturwissenschaften und Theologie als Kontraste}

Unter denen, die das Verhältnis von Theologie zu den Naturwissenschaften unter dem Aspekt des Kontrasts sehen, gibt es eine starke Gruppe konservativer Vertreter, So ist Aldert van der Ziel, Professor für Electrical Engineering an der Universität von Minnesota, Mitglied der American Scientific Affiliation. Doch verweist er in seinem Buch Genesis and Scientific Inquiry auf Gerhard von Rad als seinen theologischen Gewährsmann, während er sich in The Natural Sciences and the Christian Message auf Karl Barth beruft. Nach van der Ziel kann man

10 Theodosius Dobzhansky, Henry Margenau, Hermann Joseph Muller, Filmer S. C. Northrop und Harlow Shapeley sind auch international anerkannte Fachleute auf ihren Gebieten. 
die Naturwissenschaften und den christlichen Glauben weder gegeneinander ausspielen noch miteinander harmonisieren, sondern beide ergänzen sich komplementär. Sie sind radikal verschieden und haben völlig andersartige Ziele. Das wissenschaftliche Weltbild ist durch den wissenschaftlichen Fortschritt ständig gefährdet, während das theologische Weltbild davon unbeeinflußt bleibt. Deshalb kann man nicht sagen, daß neue, wissenschaftliche Entdeckungen den christlichen Glauben erleichtert hätten. Da beide Weltbilder sich nicht beeinflussen, muß man auch etwa bei der Genesiserzählung streng zwischen der Botschaft und dem in einen antiken Rahmen gefaßten Weltbild unterscheiden. Auf Grund dieser Unterscheidung kann auch die literarkritische alttestamentliche Wissenschaft den Glauben nicht beeinträchtigen ${ }^{11}$. Nach van der Ziel ergeben sich für den christlichen Naturwissenschaftler zwei Aufgaben: Er muß in den Naturwissenschaften tatkräftig mitarbeiten und ihnen gegenüber eine positive aber kritische Haltung einnehmen. Der Kirche gegenüber ist er verpflichtet, die Mitglieder und besonders den Klerus auf die Ergebnisse und Einstellung der modernen Naturwissenschaften aufmerksam zu machen. Obwohl er somit eigentlich zur Glaubwürdigkeit oder Unglaubwürdigkeit der christlichen Botschaft als Naturwissenschaftler nichts unmittelbar beiträgt, vermittelt er nach van der Ziel doch den Hintergrund auf dem sie zum Leuchten gebracht werden muß.

Bernard Ramm, Professor für Systematische Theologie am California Baptist Theological Seminary, zeigt in seinem Buch The Christian View of Science and the Scripture oftmals eine fast biblizistische Einstellung. So versucht er etwa die Jonaerzählung historisch zu verstehen und möchte auch die Evolutionstheorie nur als Hypothese gelten lassen. Doch meint er, daß die biblische Offenbarung keine wissenschaftlichen Entdeckungen vorwegnahm, auch wenn sich beide manchmal überschneiden. Der Heilige Geist teilte den biblischen Schriftstellern nicht die Geheimnisse der modernen Naturwissenschaften mit, sondern brachte durch sie unfehlbar wahre theologische Lehren in der kulturellen Begrifflichkeit jener Zeit zur Sprache ${ }^{12}$. John W. Klotz, Professor und Vorstand der Abteilung für Naturwissenschaften am Concordia Senior College in Fort Wayne, Indiana, möchte ebenfalls die Frage einer evolutionären Entwicklung offenlassen, auch wenn ihr die meisten Naturwissenschaftler zustimmen, denn Gott kann durch Wunder den naturgesetzlichen Zusammenhang aufheben. Trotzdem versucht Klotz, die Naturwissenschaften im Rahmen einer natürlichen Theologie zu verstehen, indem er sie als Teil der natürlichen Gotteserkenntnis ansieht ${ }^{13}$.

11 Aldert van der Ziel, Genesis and Scientific Inquiry, 196.

12 Bernard Ramm, The Christian View of Science and Scripture, 136.

13 John W. Klotz, Modern Science in the Christian Life, 79. 
Martin J. Heinecken, em. Professor für Systematische Theologie am Lutheran Theological Seminary in Philadelphia, Pennsylvania, schlägt hingegen vor, daß man die Bibel als das ansehen soll, was sie wirklich ist, als ein interpretierendes Zeugnis der Selbstmitteilung des in einer Folge von Ereignissen handelnden Gottes, die im Leben, Sterben und Auferstehen Jesu als des Christus gipfeln, und nicht als ein Textbuch der Naturwissenschaften, der Geschichte und der Geographie. Unter dieser Voraussetzung kann es keinen Konflikt zwischen den Ergebnissen der Naturwissenschaften und den Behauptungen der Offenbarung Gottes geben. Verläßt jedoch der Naturwissenschaftler oder der Theologe seinen jeweils eigenen Forschungs- und Zuständigkeitsbereich, so ergeben sich Konflikte. In ähnlicher Weise will Eric C. Rust, Professor für christliche Philosophie am Southern Baptist Theological Seminary in Louisville, Kentucky, zwischen Theologie und Naturwissenschaften unterscheiden. Er sieht den Dialog zwischen den naturwissenschaftlichen und den theologischen Sprachen besonders dadurch erschwert, daß sie die Bereiche ihrer Zuständigkeit oft überschreiten, wie es sich etwa in der Idee von Gott als des Lückenbüßers für noch fehlende wissenschaftliche Erkenntnis ausdrückt. Rust erkennt, daß die Naturwissenschaften selektiv vorgehen und von der Gesamtheit der Wirklichkeit abstrahieren, während die religiöse Sprache behauptet, über die der natürlichen Ordnung zugrundeliegende personale Tiefe Aussagen zu machen, und so die Natur in grundlegender Weise zu erfassen. Die beiden Sprachweisen bewegen sich also nicht auf gleicher Ebene. Während die theologische Sprache die naturwissenschaftliche umfassen und deuten kann, ist das im umgekehrten Fall nicht möglich, da sich die Naturwissenschaften nur mit dem empirisch Beobachtbaren befassen. Die theologische Sprache beschreibt somit die Gesamtheit der Wirklichkeit, während die naturwissenschaftliche Sprache nur eine Teilschau bietet. Außerdem wird in der Theologie das Universum in personalen Kategorien beschrieben, während die Naturwissenschaften vom Persönlichen abstrahieren und sich mit dem Unpersönlichen befassen ${ }^{14}$. Durch die Konzentration der Theologie auf das Personale fällt auch der herkömmliche Transzendenzbegriff und Rust versteht unter Transzendenz $z$ wischenpersonale Verhältnisse und Selbst-Transzendenz.

Auch der englische Physiker und Bibliothekar G. D. Yarnold möchte die naturwissenschaftliche Erklärung wvon unten* durch eine weitere und tiefere Interpretation "von oben « ergänzt sehen ${ }^{15}$. Obwohl er meint, daß das Zusammenfließen von Symbolischem, Legendärem und Objektivem in der Bibel ihre Interpretation äußerst erschwert, möchte er doch

14 14. Eric C. Rust, Science and Faith, 129.

15 G. D. Yarnold, The Spiritual Crisis of the Scientific Age, 7. 
das leere Grab, die gefalteten Grabtücher und einige, falls nicht alle, nachösterlichen Erscheinungen als objektive Fakten verstehen ${ }^{16}$. Ahnlich möchte der 1960 verstorbene reformierte schottische Theologe John Baillie Naturwissenschaften und Theologie klar gegeneinander abgrenzen und gleichzeitig die Priorität der Theologie behaupten. Die Begriffskategorien der Naturwissenschaften können nach Baillie nicht auf das innere Leben angewendet werden, ohne das Humanum zu gefähr$\operatorname{den}^{17}$. Wir sind Menschen bevor wir Wissenschaftler sind, und nur im Kontext der wahren Menschlichkeit haben die Naturwissenschaften ihren Platz. Das heißt nach Baillie nicht, daß nicht auch der Glaube für naturwissenschaftliche Belehrung aufgeschlossen sein muß. Jedoch müssen sich die Naturwissenschaften den umfassenderen Behauptungen des geistigen Lebens beugen.

Edward LeRoy Long, Jr. Professor für Religion am Oberlin College in Ohio, zeigt in seiner aufschlußreichen Studie Religious Beliefs of American Scientists, daß die einfache Tatsache, daß man Naturwissenschaftler ist, nicht schon von sich aus zu einer bestimmten Glaubenshaltung verpflichtet. Naturwissenschaftler in Amerika zeigen bei Glaubensüberzeugungen dieselbe Streubreite wie die Gesamtbevölkerung ${ }^{18}$. Nach Long stehen Naturwissenschaften und Religion in einem dialektischen Verhältnis. Nur wenn eine fähige Naturwissenschaft naturwissenschaftliche Sachverhalte behandelt und eine kompetente Religion religiöse Sachverhalte, und wenn ein dialektischer Versuch, der beide zu einem ganzheitlichen Weltbild in Beziehung bringt, die Kluft zwischen beiden überwindet, kann es zu einer wahren Versöhnung zwischen beiden kommen. Long betrachtet die Welt des Glaubens aber nicht der der Naturwissenschaften überlegen, obwohl er meint, daß es religiöser Einsicht bedarf, um das Gefühl der Furcht (awe), das die Naturwissenschaften in uns erregen, mit positivem Inhalt zu füllen. Naturwissenschaften und Religion haben komplementäre Funktion und es bedeutet keinen Fortschritt, wenn man beide zu vermischen sucht, denn keine von beiden kann die Funktion der anderen erfüllen. Wenn sich beide aber nicht um Zusammenarbeit bemühen, dann kann nach Long die ganze Wahrheit nicht voll erkannt werden, da uns Naturwissenschaften und christlicher Glaube nicht die gleiche Wahrheit geben.

M. Holmes Hartshorne, Professor für Philosophie und Religion an der Colgate Universität in Rochester, New York, kommt ähnlich wie Long zum Ergebnis, daß die Naturwissenschaften der Wahrheit der Religion nicht widersprechen, sondern sich überhaupt nicht mit ihr befas-

16 G. D. Yarnold, a. a. O., 132.

17 Zum Folgenden vgl. John Baillie, Natural Science and the Spiritual Life, $42 \mathrm{f}$.

18 Edward LeRoy Long, Jr., Religious Beliefs of American Scientists, $145 \mathrm{f}$. 
$\operatorname{sen}^{19}$. Naturwissenschaftliche Erkenntnis ist auf das Gefüge von Ursache und Wirkung beschränkt und schließt nicht die die Religion betreffenden Kategorien von Freiheit und geschichtlicher Bedeutung ein ${ }^{20}$. Gottes Wirken ist daher nicht in der Natur ersichtlich, sondern nur durch die Natur hindurch. Hartshorne erkennt, daß der christliche Glaube an Gott den Schöpfer zu einer Säkularisierung der Natur führte, wodurch die Naturwissenschaften ermöglicht wurden, die allmählich Gott den Schöpfer aus dem Blickfeld verdrängten. So entstand die Überzeugung, daß der Mensch Gewalt über die Natur hat und sie ohne Sünde kontrollieren kann. Dieser Optimismus ist nach Hartshorne eine wichtige Wurzel des naturwissenschaftlichen Fortschrittsglaubens. Damit kommt Hartshorne zu dem Schluß, daß der Konflikt zwischen Theologie und Naturwissenschaften nicht so sehr ein Konflikt zwischen Glaube und Unglaube ist, sondern zwischen zwei entgegengesetzten Glaubenshaltungen $^{21}$, dem Glauben an den Menschen als eines zum Guten befähigten Wesens und dem Glauben an Gott durch Christus.

Weniger pessimistisch ist John Dillenberger, Dekan und Professor für Historische Theologie an der Graduate Theological Union in Berkeley, Kalifornien, in seiner gründlichen historischen Analyse Protestant Thought and Natural Science. A Historical Interpretation. Nach ihm sind die neuen Naturwissenschaften nicht mehr von einer Metaphysik umgeben, deren Existenz sich lange Zeit unheilvoll für die Theologie auswirkte. Naturwissenschaftliche Begriffe können somit nicht mehr als Ausgangspunkt für religiöse Ideen benutzt werden, noch sollte die Religion naturwissenschaftliche Lücken zu schließen versuchen. Obwohl Dillenberger von einer Analogie oder Komplementarität zwischen beiden Bereichen nichts wissen will, darf man sie nach ihm auch nicht radikal trennen. Ihre jeweils verschiedenen Objektsbereiche haben nicht zur Folge, daß die Folgerungen aus einem Bereich im anderen übergangen werden können. Jedoch darf das, was in einem Bereich geschieht, nicht für den wesentlichen Inhalt des andern bestimmend werden ${ }^{22}$. So beruht ein christliches Weltverständnis immer auf einer besonderen Geschichte und auf einem besonderen Geschehen in der Geschichte, nämlich Jesus Christus. Die für theologische Aussagen zentrale Bedeutung des Christusereignisses, die bei Dillenberger sichtbar wird, wird bei den Theologen und Naturwissenschaftlern besonders betont, die das Verhältnis von Theologie und Naturwissenschaften vornehmlich unter dem Aspekt des Kontrastes sehen. Dies verändert sich jedoch sofort, wenn wir uns den Vertretern der zweiten Hauptgruppe zuwenden, die Theologie und

\footnotetext{
19 M. Holmes Hartshorne, The Promise of Science and the Power of Faith, 116.

20 M. Holmes Hartshorne, a. a. O., 132.

21 M. Holmes Hartshorne, a. a. O., 127.

22 John Dillenberger, Protestant Theology and Natural Science, $289 \mathrm{f}$.
} 
Naturwissenschaften als Parallelbewegungen betrachten. Dort hat nämlich der Glaube an Gott zentrale theologische Bedeutung.

\section{II}

\section{Naturwissenschaften und Theologie als Parallelbewegungen}

Werden Naturwissenschaften und Theologie als parallellaufende Bewegungen verstanden, so kann man unter den Vertretern dieser Interpretationsweise meist zwei Gruppen unterscheiden, die Vertreter der herkömmlichen liberalen Theologie und die der Process-Theologie.

Der geistige Vater der Process-Theologie, der englische Mathematiker und Philosoph Alfred North Whitehead ( $\dagger$ 1947), hat sich selbst ausführlich mit dem Verhältnis der Theologie zu den Naturwissenschaften beschäftigt. Schon 1925 erklärte er, daß der Verlauf der Geschichte davon abhängt, wie unsere Generation das Verhältnis zwischen Theologie und Naturwissenschaften bestimmt ${ }^{23}$. Whitehead erklärt die fortdauernden Konflikte zwischen beiden dadurch, daß er auf die kontinuierliche Entwicklung verweist, die beide durchgemacht haben. Der Zusammenstoß zwischen beiden zeigt, daß es umfassendere Wahrheiten und bessere Gesichtspunkte gibt, unter denen die Versöhnung zwischen einer tieferen Religion und einer tieferschürfenden Naturwissenschaft gefunden werden kann. Whitehead behauptet, daß wir in keinem der beiden Gebiete etwas annehmen dürfen, das nicht von der gesunden Vernunft bestätigt ist und sich auf eigene gründliche Forschung oder auf maßgebende Autoritäten stützt ${ }^{24}$. Nach Whitehead befassen sich dabei die Naturwissenschaften mit den allgemeinen Erscheinungen, die nach unserer Beobachtung die physikalischen Phänomene regulieren, während die Religion sich mit der Besinnung auf moralische und ästhetische Werte beschäftigt. Da die ewigen Prinzipien der Religion eine ständige Entwicklung ihrer Ausdrucksweise benötigen, muß diese fortlaufende Neuinterpretation nicht als Rückzug, sondern als Gewinn verstanden werden. Diese Erkenntnis ist der erste entscheidende Punkt im Gespräch mit den Naturwissenschaften. Ebenso wichtig ist auch, daß Gott nicht unter dem Aspekt der Macht gesehen wird, da das sofort eine kritische Reaktion hervorruft und zum Untergang der Religion führt ${ }^{25}$. Vielmehr muß Gott als Gott der Liebe verstanden werden, als überzeugende Macht

23 Alfred North Whitehead, Science and the Modern World, 260. Hier sollte wenigstens anmerkungsweise der in Amerika besonders einflußreiche englische Physiko-Chemiker und Philosoph Michael Polanyi erwähnt werden, dem in ähnlicher Weise an einem sich gegenseitig bedingenden Parallelismus gelegen ist.

24 Alfred North Whitehead, a. a. O., 264.

25 Alfred North Whitehead, a. a. O., 274. 
(persuasive power). Wenn wir auch an diesem zweiten Punkt umdenken können, dann, so ist Whitehead überzeugt, wird die unmittelbare Reaktion auf dieses Gottesbild Hingabe an Gott sein. Diese Hingabe wird dann von der Macht der Liebe umschlossen, deren Ziel eine ewige Harmonie ist.

Charles Hartshorne, em. Arbel Smith Professor für Philosophie an der Universität von Texas in Austin, ist der wohl bekannteste Vertreter der Process-Theologie in Amerika. In seinem Buch Man's Vision of God and the Logic of Theism nimmt er die These Whiteheads auf, daß Gott Liebe ist. Gott ist nicht der transzendente Tyrann, er ist nicht schlechthin unendlich und vollkommen, sondern in manchen Bezügen begrenzt und unvollkommen. Wegen dieser Begrenzung Gottes ist es möglich, streng allgemeine Wahrheiten zu erkennen und nicht kontingente Wahrheiten schon a priori zu wissen. Gott als die integrierte Summe unserer Existenz umfaßt notwendige und kontingente Wahrheiten. Somit umfaßt er auch die Metaphysik, die die notwendigen Aspekte unserer Existenz ergibt, und die besonderen Wissenschaften, die die kontingenten Aspekte liefern. Damit kann es nach Hartshorne letztlich keinen Konflikt zwischen Theologie und Naturwissenschaften geben.In seiner Untersuchung A Natural Theology for Our Time bestätigt er wiederum, daß man die göttliche Existenz nicht aus empirischen Gründen ablehnen kann, so daß sich daraus, etwa durch irgendwelche neue Ergebnisse der Naturwissenschaften, eine Infragestellung des Theismus ergäbe $^{26}$. In beinahe pantheistischer Ausrichtung wird Gott hier von Hartshorne als die ungeborene und unsterbende Gottheit in jedem von uns angesehen ${ }^{27}$. Hartshorne behauptet hier sogar, daß sich Gott zu jeder Kreatur wie der menschliche Geist zu den menschlichen Nervenzellen verhält. Hartshorne ist sich natürlich bewußt, daß die Erfassung des Kosmos als Nervensystem einen einzigartigen Spezialfall darstellt, den man nicht beliebig ausdeuten $\mathrm{kann}^{28}$.

Von dieser radikalen theistischen Process-Theologie unterscheidet sich Kenneth Cauthen, Professor für christliche Theologie am Crozer Theological Seminary in Rochester, New York, durch seinen christozentrischen Ansatz. Er vertritt zwar auch eine zukunftorientierte PorcessTheologie, die auf einen schöpferischen Zweck am Grund der Wirklichkeit hinweist, und genügt, eine gläubige, hoffnungserfüllte, von der Liebe bewegte Aktion zu erwecken und aufrechtzuerhalten, die die bestmögliche Zukunft herbeibringt ${ }^{29}$. Doch will er als Grundvoraussetzung seines

26 Charles Hartshorne, A Natural Theology for Our Time, 92.

27 Charles Hartshorne, a. a. O., 103.

28 Charles Hartshorne, a. a. O., 98.

20 Kenneth Cauthen, Science, Secularization \& God, 225. 
Denkens eine christozentrische Theologie der Geschichte mit einer theozentrischen Philosphie der Natur verbunden wissen. Das ergibt dann nach seinen Vorstellungen einen naturalistischen Theismus, der einen zweckvollen Kosmos zum Mittelpunkt hat, welcher im Lichte der symbolischen Bedeutung des Kreuzes und der Auferstehung Jesu von Nazareth interpretiert wird. Solch eine Schau Gottes und der Welt kann nach Cauthen der Kirche und der Welt als Lebensgrundlage für den Menschen geboten werden, der Sinn und Erfüllung sucht ${ }^{30}$.

Ian G. Barbour entfernt sich in seinem bedeutsamen Werk Issues in Science and Religion noch mehr als Cauthen von der Process-Theologie, obwohl er Whitehead häufig beifällig zitiert. Barbour findet bedeutsame Parallelen zwischen den Methoden der Theologie und der Naturwissenschaften und ist auch von der Notwendigkeit eines integrierten Weltbildes überzeugt. Er möchte selbst die Bedeutung einer Theologie der Natur verteidigen, denn, obwohl die Theologie von der geschichtlichen Offenbarung und dem Bereich der persönlichen Existenz ihren Ausgang nimmt, darf sie dort nicht stehenbleiben ${ }^{31}$. Obwohl Barbour auch wie Whitehead die Allmacht Gottes ablehnen, ist er doch bei seinen Folgerungen sehr gemäßigt, wenn er etwa anführt, daß der Mensch die Freiheit hat, die Absichten Gottes zurückzuweisen ${ }^{32}$, und daß solche Freiheit die Voraussetzung für freiwillige Liebe und für eine ungezwungene Antwort des Menschen ist. Ferner folgert er, daß nicht alles, was geschieht, Gottes Wille ist, da man die Existenz des Bösen und das tragische Element im Leben mit äußerstem Ernst bedenken muß. Barbour möchte auch in Analogie zu Whitehead die Lehren von der Schöpfung und der Vorsehung zum Begriff der ständigen Schöpfung zusammenzufassen, ohne allerdings das Verständnis unserer Abhängigkeit von Gott aufzugeben. Doch möchte er auf die Schöpfung aus dem Nichts als ursprünglicher In-Akt-Setzung verzichten, da Gottes Voraussein auch ohne sein Voraussein in der Zeit ausgesagt werden kann. Ob Gott damit nicht von der schöpferischen Urkraft zu einer Kraft in der Schöpfung und damit zu etwas Göttlichem degradiert wird, sei hier gegen Barhour nur am Rande vermerkt.

Schließlich müssen wir noch auf Langdon Gilkey, Professor für Theologie an der Divinity School der Universität von Chicago, verweisen. Auch für ihn ist der Anknüpfungspunkt zwischen einer rationalen, technologischen Welt und dem christlichen Glauben von großer Wichtigkeit. Obwohl nach Gilkey in unserer säkularen Welt die kosmischen Mythen am Vergehen sind, entdeckt er, daß dennoch Mythen weiter-

\footnotetext{
30 Kenneth Cauthen, a. a. O., 9.

31 Ian G. Barbour, Issues in Science and Religion, 5.

32 Zum Folgenden vgl. Ian G. Barbour, a. a. O., $457 \mathrm{f}$.
} 
geführt werden, auch wenn sie jetzt meist nur vom Menschen und dessen Macht handeln ${ }^{33}$. Zwei mythische Motive spielen in unserer Zeit eine besonders große Rolle, der Mythos von der unendlichen Möglichkeit, das Geheimnis aller Dinge zu wissen, und der damit verwandte Mythos, daß der Mensch die Mächte, die er jetzt kennt, kontrollieren kann ${ }^{34}$. Doch behauptet Gilkey, daß der Mythos vom Menschen, der durch $\mathrm{Zu}$ wachs an Wissens mündig geworden ist, nicht nur ein inakkurater theologischer Mythos ist. Er ist sogar ein gefährlicher Mythos in den angewandten Wissenschaften ${ }^{35}$, denn der Mensch zeigt sich selbst dort im paradoxen Gewand des entweder frei Untersuchenden oder des determinierten Untersuchten. In dieser paradoxen Situation von Freiheit und Determinismus, die sich etwa im Krankenhaus in der Paradoxie von »allwissendem* Arzt und »hilflosen « Patienten ausdrückt, bedarf der Mensch zur Hoffnung und Gewißheit, wie in jedem anderen Zeitalter, eines diese Paradoxien transzendierenden Herrn aller Dinge ${ }^{\mathbf{3 6}}$.

Nun müssen wir uns noch dem weiten Feld der liberalen Theologie zuwenden, soweit sie sich an dem Gespräch mit den Naturwissenschaften beteiligt. Auch hier wollen wir nur einige Schlaglichter aufwerfen. Harold Schilling, Professor der Physik und Dekan der Graduate School an der Pennsylvania State University sieht eine große Ahnlichkeit zwischen Religion und den Naturwissenschaften. Beide bestehen aus drei Komponenten, dem Empirischen, dem Theoretischen und dem Umformenden. Jede dieser drei Komponenten ist von den beiden anderen abhängig, jedoch ist keine die logische Grundlage der anderen. Dadurch ergibt sich der zirkuläre und wesentlich unlogische Charakter der beiden Gebiete. Schilling sieht in beiden Gebieten gleiche Kräfte walten. Damit stehen sich die beiden Bereiche nicht grundsätzlich feindlich gegenüber. Sie verhalten sich komplementär, indem sie jeweils dem anderen Bereich ihre einzigartigen Einsichten zukommen lassen, und sie sind beide unersetzlich, um volle Einsicht und Glauben $\mathrm{zu}$ erlangen und in angemessener Weise mit den schwierigen Problemen fertigzuwerden, die uns begegnen ${ }^{37}$.

Besonders in den John Calvin McNair Lectures betont C. A. Coulson, Rouse Ball Professor der Mathematik in Oxford, England, und methodistischer Laienprediger, die natürliche Erkennbarkeit Gottes. Die Naturwissenschaften sind nach Coulson eine Offenbarung Gottes, die mit der Betonung der Werte und der Person im herkömmlichen christlichen Glauben im Einklang stehen und ihm zudem noch gewisse Ele-

\footnotetext{
3s Langdon Gilkey, Religion and the Scientific Future, 76.

s4 Langdon Gilkey, a. a. O., 79.

85 Langdon Giley, a. a. O., 95.

36 Langdon Gilkey, a. a. O., 100.

37 Harold K. Schilling, Science and Religion, 247.
} 
mente hinzufügen, die wir sonst nicht erreichen könnten ${ }^{38}$. Dabei sind Größe und Grausamkeit der Natur gleichermaßen Offenbarung Gottes. Während die Naturwissenschaften eine Abstraktion eines umfassenderen Bereichs der Wirklichkeit bieten, ist die Religion die totale Antwort des Menschen an seine ganze Umgebung. Deswegen ist es für den Christen nötig, etwa zu erkennen, was sich in der neuen industriellen Revolution abspielt, damit er zeigen kann, daß die dort begegnenden Probleme nicht rein technologischer Art sind. So kann der Christ ein Bezugssystem liefern, auf das Entscheidungen und Handlungen bezogen werden können $^{39}$. Trotz der natürlichen Gotteserkenntnis ist Coulson überzeugt, daß wir Christus benötigen, damit alle Natur als heilig verstanden werden kann und in aller Unruhe und Verwirrung Gottes Vollkommenheit anwachsen $\mathbf{k a n n}^{40}$. Obwohl uns die Naturwissenschaften die Größe des Raumes und der Zeit und die seltsame Harmonie der Dinge zeigen, genügt diese Erkenntnis allein nicht. Coulson versteht zwar alles Leben sakramental und propagiert die Notwendigkeit der Natur zu einem rechten Verstehen Christi, doch schränkt er gleichzeitig ein, daß allein ein Christ die säkulare Welt zur Hoffnung befähigen kann ${ }^{41}$.

Coulson beruft sich oft auf Charles E. Raven, dem ehemaligen Regius Professor für Divinity in Cambridge, England, der ihm in der Betonung der Einheit von Natur und Religion nahesteht. Nach Raven ist die religiöse Erfahrung ein grundlegendes und allgemeingültiges Charakteristikum des Menschen. Gott, der in der Erfahrung des Mysteriums wahrgenommen wird, offenbart sich als Quelle und Grund der Existenz des Universums, als Wirklichkeit und Archetypus seiner Struktur, als verbindende Kraft, die seine Entwicklung in Gang setzt und vorantreibt ${ }^{42}$. Natur und Ubernatur dürfen also nicht wie in der Neuorthodoxie voneinander getrennt werden, da man sonst in ein arianisierendes Fahrwasser gerät ${ }^{48}$. Wenn Gott ist und sich in Christus offenbart hat, dann sind Schöpfung, Erlösung und Heiligung nach ihrem Ursprung identisch und haben gleichen Charakter. Gnade darf somit nicht in einem radikalen Kontrast zur Schönheit, Wahrheit und Güte der natürlichen Ordnung gesehen werden, da solches den Glauben an eine wirkliche Inkarnation ausschließen würde. Damit hat der ganze Evolutionsvorgang einen zweckgerichteten Drang, der nicht nur seinen Betätigungsbereich ständig erweitert, die Individualität vervollkommnet, und schließlich das Persönliche hervortreten läßt, sondern auch eine Harmo-

\footnotetext{
$\$$ C. A. Coulson, Science and Christian Belief, 3.

C. A. Coulson Science, Technology and the Christian, $108 \mathrm{f}$.

10 C. A. Coulson, Science and Christian Belief, $117 \mathrm{f}$.

1 C. A. Coulson, Science, Technology and the Christian, 110.

42 Charles E. Raven Science, Religion and the Future, 118.

4s Charles E. Raven, Natural Religion and Christian Theology, Bd. I, 2.
} 
nie der Vielfalt, die das allmähliche Erfüllen eines Planes und den Einbau der verschiedenen Elemente des Entwurfs in ein komplexes und einschließendes Ganzes vorantreibt ${ }^{44}$.

Diese fast monistische Ausrichtung im Gespräch zwischen Theologie und den Naturwissenschaften zeigt sich noch verstärkt bei Arnulf K. Esterer. Nach Esterer bieten Religion und die Naturwissenschaften nur verschiedene Aspekte des gleichen Universums. Durch diese Zusammenschau hofft er, die traditionelle Kluft $z$ wischen beiden überwinden zu können. Unser Geist ist Teil der geistigen Welt, die der Materie ihre Richtung gibt ${ }^{45}$. Materie und Geist sind somit miteinander verflochten, so daß wir an der Welt mitbauen können. Auch hängt es von uns ab, ob wir uns nach unseren besten Möglichkeiten verbessern, da unsere Einstellung und unsere Taten uns überdauern. Esterer lehnt den Gedanken einer letzten Abrechnung $a b$, da es nach seiner Öberzeugung nur eine ständige Anhäufung unseres Verhaltens ergibt, weil unsere Vergangenheit und unsere Zukunft Teil unser selbst sind.

Der englische Geologe und Geochemiker und anglikanische Geistliche Arthur F. Smethurst kann nicht im eigentlichen Sinn als liberal bezeichnet werden. Obwohl inhaltsweise seine Aussagen konservativ anmuten, kann man nicht umhin, seine Argumentationsweise liberal zu nennen, da er versucht, wie besonders bei der Behandlung der Wunder deutlich wird, Glaubensinhalte rational einsichtig zu machen. Wir wollen ihn deshalb hier nur anhangsweise anführen. Smethurst möchte den christlichen Glauben mit den Naturwissenschaften so verknüpfen, daß er die Grundlage für die Naturwissenschaften abgibt. Smethurst sieht nämlich in der wissenschaftlichen Methode drei grundlegende Glaubensüberzeugungen ausgedrückt, den Glauben an eine Ordnung im Universum, den Glauben an das Kausalitätsprinzip oder an die Einsichtbarkeit der natürlichen Welt und den Glauben an die Zuverlässigkeit der menschlichen Vernunft. Doch können wir an die universale Gültigkeit der Ordnung und an die Stetigkeit und Rationalität des Universums nur dann glauben, wenn wir annehmen, daß die materielle Welt das Werk eines einzigen, rationalen und göttlichen Geistes ist ${ }^{46}$. Unsere wissenschaftliche Haltung gegenüber dem Universum und die Entwicklung der wissenschaftlichen Methode konnte sich also nach Smethurst allein in einer intellektuellen Umgebung ergeben, in der der maßgebende religiöse Glaube monotheistisch ausgerichtet und von dem Sein eines einzigen, rationalen, göttlichen Wesens überzeugt ist ${ }^{47}$. Fällt dieser Glaube

"Charles E. Raven, a. a. O., Bd. II, 146.

45 Zum Folgenden vgl. Arnulf K. Esterer, Towards a Unified Faith, $99 \mathrm{f}$.

46 Arthur F. Smethurst, Modern Science and Christian Belief, 13.

47 Arthur F. Smethurst, a. a. O., 14. 
weg, bricht das Fundament der wissenschaftlichen Methode in sich zusammen.

\section{III}

\section{Naturwissenschaften als Ausgangspunkt der Theologie}

Von der Behauptung, daß Naturwissenschaften und Theologie parallelgerichtete Bewegungen sind, ist es nur ein kleiner Schritt zur Annahme, daß die Naturwissenschaften als Ausgangspunkt für theologische Bemühungen dienen können. Auch hier gibt es eine große Streubreite der Richtungen, von den Theisten und Deisten, die sich noch im Bereich des christlichen Glaubens zu bewegen wähnen, bis hin zu den extremen Naturalisten, die den Gottesbegriff selbst aufgegeben haben.

William G. Pollard behauptet in fast neuorthodoxer Weise, daß es einen zweifachen Charakter der Wirklichkeit gibt, den die Naturwissenschaften auf Grund ihres Wesens nicht einsehen können. So erstreckt sich die Wirklichkeit vor uns in zwei verschiedenen und scharf voneinander getrennten Dimensionen oder Welten ${ }^{48}$. Die biblische Gedankenwelt ist vorwiegend, wenn auch nicht ausschließlich, mit der einen dieser Dimensionen beschäftigt, während die Naturwissenschaften als solche, aber nicht die Beschäftigung mit ihnen, ausschließlich auf den anderen Bereich begrenzt ist. Der Mensch vereinigt nun beide Bereiche in sich und bewegt sich in beiden analog, denn es gibt keinen völlig freien Intellekt, der in einem einsamen und isolierten Selbst tätig ist ${ }^{\mathbf{4 9}}$. Treibt man das Geschäft der Naturwissenschaften, dann muß man sich ihnen völlig hingeben, treibt man das Geschäft der Theologie, dann muß man sich Christus und seiner Kirche völlig und frei widmen. Die $\mathrm{Zu}$ fälligkeit der Natur begreift jedoch das in sich, was die Zufälligkeit dieser Bereiche transzendiert, nämlich die Existenz und Realität der Ubernatur. Dennoch teilt sich unsere menschliche Wirklichkeitserfahrung, wie die ganze Menschheitsgeschichte bezeugt, in eine begriffliche und eine nichtbegriffliche Komponente ${ }^{50}$. Erstere umschließt das Gebiet der Naturwissenschaften und hat das Verständnis der Natur zum Objekt. Letztere beinhaltet das Gebiet der Religion und der Dichtkunst und hat das Verständnis der Ubernatur zum Gegenstand. Jedoch ist diese Teilung unserer Wirklichkeitserfahrung nicht durch die Wirklichkeit als ganzer präformiert, sondern sie ist Ausdruck unserer beschränkten Erfahrung.

\footnotetext{
48 Vgl. zum Folgenden William G. Pollard, Chance and Providence, 154.

40 William G. Pollard, Physicist and Christian, 23.

so William G. Pollard, a. a. O., 110.
} 
Der verstorbene Astronom und Physiker Sir Arthur Eddington schlug in seinem einflußreichen Buch The Nature of the Physical World eine ähnliche Trennung vor. Nach Eddington können Religion und Naturwissenschaften nicht im Konflikt miteinander stehen. Wenn beide sich auf ihr eigentliches Gebiet beschränken, kann es nur an der gemeinsamen Grenze zu Meinungsverschiedenheiten kommen. Für beide ist der Ausgangspunkt ihrer Überlegungen die Überzeugung von der Bedeutung dessen, was man sich bewußt wird. Somit will Eddington nicht die Glaubensinhalte der Religion mit den Daten oder Methoden der Naturwissenschaften bweisen ${ }^{51}$. Doch scheint er den christlichen Rahmen $\mathrm{zu}$ verlassen, wenn er eine mystische Religion vorschlägt, die auf einer Erfahrung beruht, der man sich selbst bewußt wird und als grundlegend anerkennt. Da Religion und Naturwissenschaften nach Eddington gleiche Grundlage haben, nämlich, das was man sich selbst bewußt wird, können sie miteinander in ein Gespräch eintreten.

Theodosius Dobzhansky, Professor für Genetik an der Universität von Kalifornien in Davis, unterscheidet nicht mehr wie Eddington zwischen zwei Erfahrungsbereichen, sondern für ihn ist Religion eine menschliche Notwendigkeit. Nach Dobzhansky ist Selbstgewahrsein eines der grundlegenden und vielleicht das grundlegende Charakteristikum des Menschen ${ }^{52}$. Doch mit dem Selbstgewahrsein kam über den Menschen auch Furcht, Angst und Todesgewahrsein. Deshalb braucht der Mensch Glaube, Hoffnung und Zweck, um zu leben und seiner Existenz Bedeutung und Würde zu geben, denn er ist die einzige lebende Spezies, die eine solch höchste Beunruhigung verspürt ${ }^{53}$. Er allein erkennt, daß er nicht durch eigene Wahl in dieser Welt ist und er sehnt sich nach Liebe und Angenommensein. Fruchtbarkeitskulte und die elementare Freude des Am-Leben-seins reichten aus, um den primitiven Völkern wenigstens einen Schein des Daseinssinnes zu geben. Doch die menschliche Entwicklung hat uns darüber hinausgewiesen, so daß wir uns nicht mehr mit den primitiven Freuden des Úberlebens und der Fortpflanzung begnügen können. Deshalb braucht nach Dobzhansky der moderne Mensch eine religiöse Synthese. Das heißt nicht, daß er die bestehenden Religionen neu zum Leben erwecken kann oder daß er eine neue Religion braucht. Die Synthese kann auf der Grundlage einer oder mehrerer der großen Weltreligionen geschehen und sie muß auch die Naturwissenschaften in sich fassen. Außerdem muß sie ästhetisch zufriedenstellend und rational überzeugend sein und die ganzen Bereiche des Lebens und

51 Sir Arthur Eddington, The Nature of the Physical World, 333.

52 Theodosius Dobzhansky, The Biology of Ultimate Concern, 68.

ss Zum Folgenden vgl. Theodosius Dobzhansky, a.a. O., $108 \mathrm{f}$. Bei seiner Betonung der Notwendigkeit einer religiösen Synthese scheint Julian Huxleys Idee eines »noetic integrator* Pate gestanden zu haben. 
Nichtlebens einschließen und in ihrer wechselseitigen Aufeinanderbezogenheit bedenken. Erst dann kann der Hunger des Menschen nach Sinn des Lebens und der Welt befriedigt werden. Obwohl Dobzhansky von Tillich und von Teilhard de Chardin beeinflußt ist, lehnt er einen Gott der Gnade und der Liebe ab, da sich ein solcher nicht mit der Existenz des Übels und dem evolutionären Indeterminismus vereinbaren läßt.

Fast entgegengesetzt, doch auch von einem monistischen Weltverständnis aus argumentiert Edmund W. Sinnott, ehemaliger Professor für Botanik und Dekan der Graduate School an der Yale Universität. Er sieht Gott als das organisatorische Prinzip in der Welt an, das sich im Leben widerspiegelt ${ }^{54}$. Dieses Prinzip der Organisation schließt den Glauben ein, daß in der Welt etwas tätig ist, das zum Geist führt und selbst Geist ist. Leben ist seinem Wesen nach zweckgerichtet und schöpferisch, so daß wir in ihm Zugang zu etwas Integrierenden, Zweckgerichteten und Schöpferischen haben ${ }^{55}$. Was aber das Leben selbst ist und woher es seine organisierende Kraft hat, wissen wir nicht. Doch ist das nicht der Ubberzeugung abträglich, daß man Geist und Körper durch den Begriff des lebenden Organismus als eine Einheit erfaßt, und daß damit der menschliche Geist seinen Ursprung im Leben selbst hat. So kommt nach Sinnott der Geist aus dem Leben und ist unterwegs zum höchsten Ausdruck des Lebens, zu Gott.

Auch der verstorbene Physiker und Nobelpreisträger Arthur $\mathrm{H}$. Compton erachtet Gott als das verbindende Prinzip, das in der Natur tätig ist ${ }^{56}$. Doch will er nicht, wie Sinnott es teilweise tut, die Notwendigkeit Gottes aus der Natur ableiten, sondern er meint, daß der Glaube an einen weisen Gott wahrscheinlich auf der Erfahrung beruht, daß die Hypothese Gott eine vernünftigere Erklärung der Welt abgibt als andere Versuche, und vielleicht auch darauf, daß sie sich auf das Gemütsleben des religiösen Menschen vorteilhaft auswirkt ${ }^{57}$. Wenn aber wirklich die Erschaffung intelligenter Wesen ein Hauptziel des Schöpfers des Universums ist und wenn, wie wir wohl annehmen können, die Menschheit seine höchste Entwicklung in dieser Richtung darstellt, dann sind die Gelegenheiten und die V'erantwortung für den Menschen, als Partner Gottes an dieser großen Aufgabe mitzuwirken, fast überwältigend $^{58}$. Deshalb müssen wir in der Zusammenarbeit mit unserem Schöpfer unser Bestes geben, damit wir eine bessere Welt schaffen, in der wir leben.

\footnotetext{
s4 Edmund W. Sinnott, The Biology of the Spirit, 164. Sinnott scheint nicht ganz unbeeinflußt von Hans Drieschs Vitalismus zu sein. Er bezieht sich auch ausführlich auf Drieschs Entelechienlehre.

$5 s$ Edmund W. Sinnott, a. a. O., $167 \mathrm{ff}$.

so Arthur H. Compton, The Human Meaning of Science, 62.

57 Arthur H. Compton, a. a.O., 66.

38 Arthur H. Compton, a. a. O., 88.
} 
E. L. Mascall, Professor für Historische Theologie am King's College in London soll mit seinem Bampton Lectures von 1956 hier noch anhangweise vermerkt werden ${ }^{59}$. Obwohl er im christlichen Kontext zu verstehen ist, da er z. B. für die Jungfrauengeburt eintritt und die Supernaturalisation des Menschen in Christus als eine Folge der Inkarnation behauptet, plädiert er für einen christlichen Theimus. Vor einem Gespräch mit den Naturwissenschaften braucht man sich nach Mascall nicht zu scheuen, da beide Gebiete autonom sind und nur die metaphysischen Voraussetzungen der Naturwissenschaften mit dem christlichen Glauben in Konflikt kommen. Ob die Welt von einem Geist geschaffen wurde, oder ob der Geist von der Welt geschaffen wurde, oder beide von Gott, ist eine Frage, die jenseits des Zustandsbereichs der Naturwissenschaften liegt. Weder können kosmologische Theorien letzte theologische Relevanz haben, noch kann etwa die moderne Neurophysiologie die christliche Lehre vom Menschen erschüttern, sie kann sie nur verdeutlichen. Naturwissenschaftliche Modelle, ob sie auf physikalischen Eindrücken oder auf rein mathematischen Begriffen beruhen, sind nämlich deduktiv gewonnene Systeme, deren alleinige Aufgabe es ist, die empirischen Beobachtungen zu koordinieren und vorauszusagen. Da Mascall trotz dieser Beschränkung des Geltungsbereichs der Naturwissenschaften die Philosophie bemüht, die Behauptung der menschlichen Seele und der Unsterblichkeit zu untermauern ${ }^{60}$ scheint sich der Verdacht zu festigen, daß er neo-thomistischem Denken nahesteht.

Abschließend müssen wir uns den humanistischen Vertretern eines Gesprächs zwischen Naturwissenschaften und Theologie kurz zuwenden. So sieht etwa der ehemalige englische Mathematiker und Philosoph Bertrand Russell den Konflikt $\mathrm{zwischen}$ beiden weitgehend als einen Streit zwischen Autorität und Beobachtung61. Der Grund hierfür ist, daß man lange Zeit gewisse Glaubenbekenntnisse mit bestimmten Konfessionen und mit moralischen Konventionen verband ${ }^{62}$. Doch besteht nach Russell Religion eigentlich nur aus einer bestimmten Weise des Fühlens und nicht aus einem bestimmten Gaubenskodex. Somit kann es keinen Streit zwischen Religion und den Naturwissenschaften geben, denn die Religion hat es mit Werten zu tun, bei denen es keine Wahl zwischen wahr und falsch geben kann, während die Naturwissenschaften sich mit Wissen befassen $^{63}$. Religion und Naturwissenschaften sind daher nur zwei verschiedene Aspekte des sozialen Lebens des Menschen.

30 Vgl. E. L. Mascall, Christian Theology and Natural Science.

0 E. L. Mascall, a.a. O., 269 ff. Mascalls thomistische Ausrichtung wird besonders deutlich in seinem Buch He Who Is. A Study in Traditional Theism. The Existence of God and His Relationship to the World, London (Libra Book), (1943), 1966.

-1 Bertrand Russell, Religion u. Science, 16.

-2 Bertrand Russell, a. a. O., 9.

os Bertrand Russell, a. a. O., $242 \mathrm{f}$. 
Weit mehr Bedeutung als Russell mißt der englische Biologe und Anthropologe Julian Huxley der Religion bei64. Für ihn besteht die Natur aus einem zusammenhängenden Evolutionsprozeß, in dem es keine Stetigkeit, sondern nur richtungsweisenden Wechsel gibt. Der Mensch steht auf der dritten Stufe des Evolutionsprozesses, der psychosozialen Ebene (die anderen sind die kosmische und die biologische Ebenen), die durch die Transformation der Kultur arbeitet. Doch ist der Mensch durch drei Fragen: "Was bin ich?" "Was ist die Welt, in der ich mich befinde?" und "Was ist mein Schicksal?» verwirrt. So benötigt er einen noetischen Integrator, der in diesen Fragen richtungsweisend ist und $\mathrm{ihm}$ in seinem Entwicklungsprozeß weiterhilft. Ob dieser noetische Integrator oder dieses Glaubenssystem Ideologie oder Religion genannt wird, ist für Huxley unwichtig. Bedeutsam ist nur, daß es sicher auf den genauesten Kenntnissen aufgebaut sein muß, die der Mensch zur Verfügung hat. Diese Gedanken klingen sehr an die evolutionäre Schau Dobzhanskys an, nur daß Huxley im Gegensatz zu ihm den christlichen Einflußbereich bereits verlassen hat.

Es hat sich wahrscheinlich bei diesem kurzen Überblick schon gezeigt, daß die angelsächsische, und hier besonders die amerikanische Theologie viel zu komplex ist, um sich streng genetisch entwidkeln oder einfach in bestimmte Kategorien einordnen zu lassen. Die konfessionelle und theologische Lage ist von einem solchen Pluralismus gekennzeichnet, $\mathrm{daß}$ oft gleiche oder ähnliche Positionen an verschiedenen Orten gleichzeitig auftreten, ohne voneinander abhängig $\mathrm{zu}$ sein oder voneinander zu wissen, auch wenn sich immer mehr ein Trend zum stärkeren Aufeinanderhören abzeichnet. Was würde nun wohl Karl Heim zu dieser Vielfalt der Ansätze im Gespräch mit den Naturwissenschaften sagen? Wir können an dieser Stelle nur Vermutungen aufstellen. Es ist sicher, $\mathrm{da}$ er sich über die allerorts erkannte Notwendigkeit, Glaube und Wissen in ein bewußtes Verhältnis zueinander zu bringen, sehr freuen würde. Doch würde ihn dieses Bemühen wahrscheinlich auch in seiner Überzeugung bestärken, daß der Mensch sich über der polaren Spannungswelt, die er tagtäglichen im Beruf und auch im Glauben und Wissen erfährt, nach einer diese Polaritäten überbrückenden und in sich schließenden überpolaren Einheit sehnt. Aber er würde uns vielleicht auch zu bedenken geben, daß eine wirkliche Einheit nur im Glauben gegeben wird, nämlich im Hören auf den Sohn dessen, der die Welt als eine Einheit schuf, und der uns eine neue, alle polaren Spannungen aufhebende überpolare Schöpfung verheißt, an der wir jetzt schon in vorläufiger Form aus der im Glauben erwachsenden verantwortlichen Liebe teilhaben

64 Vgl. Julian Huxley, The Human Crisis, and *Evolution, Culture and Biology* in Yearbook of Anthropology, hg. v. W. L. Thomas, New York (Wenner-Gren Foundation), 1955. 
und sie im Umgang mit der Welt und ihren Menschen praktizieren dürfen.

\section{Einfübrendes Literaturverzeichnis}

Asimov, Isaac, The Universe. From Flat Earth to Quasar, New York (Walker), 1966.

Augenstein, Leroy, Come, Let Us Play God, New York (Harper \& Row), 1969.

Baillie, John, Natural Science and the Spiritual Life. Being the Pbilosophical Discourse Delivered before the British Association for the Advancement of Science at Edinburgh on 12 August 1951, London (Oxford University Press), 1951.

Barbour, Ian G., Christianity and the Scientist, New York (Association Press), 1960.

-, Issues in Science and Religion, Englewood Cliffs, N. J. (PrenticeHall), 1966.

-, (Hg.), Science and Religion. New Perspectives on a Dialogue, New York (Harper \& Row), 1968

-, Science and Secularity. The Ethics of Technology, New York (Harper \& Row), 1970.

Barnes, Ernest W., Scientific Theory and Religion, Cambridge (Cambridge University Press), 1933.

Bayne, Stephen F., Space Age Christianity, New York (MorehouseBarlow), 1963.

Bett, Henry, The Reality of the Religious Life. A Study of Miracle, Providence, and Prayer, New York (Macmillan), 1949.

Blum, Harold F., Time's Arrow and Evolution, Princeton, N. J. (Princeton University Press), 1951.

Barithwaite, Richard B., An Empiricist's View of the Nature of Religious Belief, Cambridge (Cambridge University Press), 1955.

Bridgman, Pency W., Reflections of a Ploysicist, New York (Philosophical Library), 1955.

Broad, Charlie D., Religion, Philosophy and Psychical Research, London (Routledge and Kegan Paul), 1953.

Bube, Richard H., (Hg.), The Encounter between Christianity and Science, Grand Rapids, Mich. (William B. Eerdmans), 1968.

Butterfield, Herbert, The Origins of Modern Science, 1300-1800, London (G. Bell and Sous), 1953.

Caillet, Emile, The Recovery of Purpose, New York (Harper), 1959.

Caldin, E. F., The Power and Limits of Science. A Philosophical Study, London (Chapman \& Hall), 1949.

-, Science and Christian Apologetic, London (Bladkfriars), 1951. 
Cauthen, Kenneth, Science, Secularization, E God. Towards a Theology of the Future, Nashville (Abingdon Press), 1969.

Clark, Cecil H.D., The Scientist and the Supernatural, London (Epworth Press), 1966.

Clark, Robert E. D., Cbristian Belief and Science. A Reconciliation and a Partnership, Philadelphia (Muhlenberg Press), 1961.

-, The Universe: Plan or Accident? The Religious Implications of Modern Science, Philadelphia (Muhlenberg Press), 1961.

Compton, Arthur H., The Human Meaning of Science, Chapel Hill (University of North Carolina), 1940.

Coulson, Charles A., Christianity in an Age of Science, New York (Oxford University Press), 1953.

-, Science and Christian Belief, Chapel Hill (University of North Carolina), 1955.

-, Science, Technology and the Christian, New York (Abingdon), 1960.

Dampier, Sir William C., A History of Science and its Relations with Philosophy and Religion, London (Cambridge University Press), $1966^{4}$.

Dillenberger, John, Protestant Thought and Natural Science. A Historical Interpretation, Garden City, N. J. (Doubleday), 1960.

Dobzhansky, Theodosius, The Biology of Ultimate Concern, New York (The New American Library), 1967.

-, Mankind Evolving. The Evolution of the Human Species, New Haven (Yale University Press), $1967^{10}$.

Eddington, Sir Arthur S., The Nature of the Physical World, Ann Arbor, Mich. (University of Michigan), (1928), 1958.

-, The Philosophy of Physical Science, Cambridge (Cambridge University Press), 1939.

Eiseley, Loren, The Firmament of Time, New York (Ateneum), 1960. Esterer, Arnulf K., Towards a Unified Faith, New York (Philosophical Library), 1963.

Filson, Joseph E., The Christian Hope. The Presence and the Parousia, London (Longmans, Green and Co.), 1954.

Gamow, George, Matter, Earth and Sky, Englewood Cliffs, N. J. (Prentice-Hall), 1958.

Gidon, Gottlieb, The Logic of Choice. An Investigation of the Concepts of Rule and Rationality, New York (Macmillan), 1968.

Gilkey, Langdon, Maker of Heaven and Earth. A Study of the Christian Doctrine of Creation, Garden City, N. J. (Doubleday), 1959.

-, Naming the Whirlwind: The Renewal of God-Language, Indianapolis (Bobbs-Merrill), 1969. 
-, Religion and the Scientific Future. Reflections on Myth, Science, and Theology, New York (Harper), 1970.

Gillispie, Charles C., Genesis and Geology. A Study in the Relations of Scientific Thought, Natural Theology, and Social Opinion in Great Britain: 1790-1850, Cambridge, Mass. (Harvard University Press), 1951.

Greene, John C., Darwin and the Modern World View, Baton Rouge (Louisiana State University Press), 1961.

-, The Death of Adam: Evolution and its Impact on Western Thought Ames (Iowa State University Press), 1959.

Haber, Francis D., The Age of the World: Moses to Darwin, Baltimore (John Hopkins Press), 1959.

Hardy, Alister D., The Divine Flame. Gifford Lectures 1964-65, Lonon (Collins), 1966.

Hartshorne, Charles, Man's Vision of God and the Logic of Theism, Chicago (Willett, Clark \& Co.), 1941.

-, A Natural Theology for Our Time, La Salle, Ill. (Open Court), 1967.

Hartshorne, M. Holmes, The Promise of Science and the Power of Faith, Philadelphia (Westminster), 1958.

Haselden, Kyle, and Hefner, Philip, (Hg.), Changing Man: The Threat and the Promise, Garden City, N. J. (Doubleday), 1968.

Hazelton, Roger, God's Way with Man. Variations on the Theme of Providence. New York (Abingdon), 1956.

Heinedken, Martin J., God in the Space Age, Philadelphia (John C. Winston Co.), 1959.

Hesse, Mary B., Science and Human Imagination, New York (Philosophical Library), 1955.

Huxley, Julian, The Human Crisis, Seattle (University of Washington Press), 1963.

-, Religion without Revelation, New York (Harper and Brothers), 1957.

Jeans, James H., Astronomy and Cosmogony, New York (Dover Publications), (1929), 1961.

-, The Universe around Us, Cambridge (Cambridge University Press), $1960^{4}$.

Kennedy, Gail, (Hg.), Evolution and Religion. The Conflict between Science and Theology in Modern America, Boston (D. C. Heath), 1967.

Klotz, John W., Modern Science in the Christian Life, St. Louis (Concordia), 1961.

Koestler, Arthur, The Sleepwalkers. A History of Man's Changing Vision of the Universe, New York (Macmillian), 1959. 
Lasker, Gabriel W., (Hg.), The Processes of Ongoing Human Evolution, Detroit (Wayne State University Press), 1960.

LeFevre, Perry D., Understandings of Man, Philadelphia (Westminster), 1966.

Lewis, John, Man and Evolution, New York (International Publishers) 1962.

Long, Edward LeRoy, Jr., Religious Beliefs of American Scientists, Philadelphia (Westminster), 1952.

-, Science \& Christian Faith. A Study in Partnership, New York (Association Press), 1950.

Lovejoy, Arthur O., The Great Chain of Being. A Study of the History of an Idea, New York (Harper Torchbooks), $1965^{5}$.

Margenau, Henry, The Nature of Physical Reality, New York (McGraw-Hill), 1950.

Martin, Charles B., Religious Belief, Ithaca, N. Y. (Cornell University Press), 1959.

Mascall, Eric L., Christian Theology and Natural Science, Hamden, Conn. (Archon Books), 1965.

-, The Openness of Being. Natural Theology Today. The Gifford Lectures 1970-1971, London (Darton, Longman \& Todd), 1971.

-, The secularization of Christianity. An Analysis and a Critique, New York (Holt, Rinehart and Winston), 1966.

Meland, Bernard E., The Realities of Faith, New York (Oxford University Press), 1962.

Meyer-Abich, Adolf, The Historic-Philosophical Background of the Modern Evolution Biology, Leiden (E. J. Brill), 1964.

Miles, Thomas R., Religion and the Scientific Outlook, London (George Allen and Unwin), 1959.

Milne, Edward A., Modern Cosmology and the Christian Idea of God, Oxford (Clarendon Press), 1952.

Mixter, Russell L., (Hg.), Evolution and Christian Thought Today, Grand Rapids, Mich. (William B. Eerdmans), 1959.

Monsma, John Clover, (Hg.), The Evidence of God in an Expanding Universe. Forty American Scientists Declare Their Affirmative Views on Religion, Published in Connection with the International Geophysical Year, New York (G. P. Putnam's Sons), 1958.

-, (Hg.), Science and Religion, Twenty-Three Prominent Churchmen Express Their Opinions, New York (G. P. Putnam's Sons), 1962.

Montagu, Ashely, Darwin, Competition E Cooperation, New York (Henry Schuman), 1952.

Munitz, Milton K., (Hg.), Theories of the Universe. From Babylonian Myth to Modern Science, New York (The Free Press), 1957.

Nagel, Ernest, The Structure of Science. Problems in the Logic of Scientific Explanation, New York (Harcourt, Brace \& World), 1961 
Niebuhr, H. Richard, Radikaler Monotheismus. Theologie des Glaubens in einer pluralistischen Welt, Gütersloh (Gerd Mohn), 1965.

Niebuhr, Reinhold, The Nature and Destiny of Man, 2 Bde, New York (Scribner), 1949.

Pelikan, Jaroslav J., The Christian Intellectual, New York (Harper \& Row), 1965).

Pike, Nelson (Hg.), God and Evil, Raedings on the Theological Problem of Evil, Englewood Cliffs, N. J. (Prentice-Hall), 1964.

Polanyi, Michael, Knowing and Being. Essays, hg. v. Marjorie Greene, Chicago (University of Chicago Press), 1969.

-, Personal Knowledge. Towards a Post-Critical Philosophy, Chicago (University of Chicago Press), 1958.

-, Science, Faith, and Society, Oxford (Oxford University Press), 1946.

Pollard, William G., Chance and Providence. God's Action in a World Governed by Scientific Law, New York (Charles Scribner's Sons), 1958.

-, Physicist and Christian; A Dialogue between the Communities, Greenwich, Conn. (Seabury Press), 1961.

Rabut, Oliver A., God in an Evolving Universe, New York (Herder and Herder), 1966.

Ramm, Bernard, The Christian View of Science and Scripture, Grand Rapids, Mich. (William B. Eerdmans), 1954.

Ramsay, Ian T., Religion and Science. Conflict and Syntbesis. Some Philosophical Reflections, London (SPCK), 1964

-, (Hg.), Biology and Personality, Oxford (Basil Blackwell), 1965.

Raven, Charles E., Christianity and Science, New York (Association Press), 1955.

-, Natural Religion and Christian Theology. The Gifford Lectures 1951, 2 Bde, Cambridge (University Press), 1953.

-, Science, Religion, and the Future, New York (Macmillan), 1943. Richardson, Alan, The Bible in an Age of Science, London (SCM Press), 1961.

-, The Miracle-Stories of the Gospels, New York (Harper \& Row), o. J.

Russell, Bertrand, Religion \& Science, New York (Oxford University Press, Galaxy Books), (1935), 1961.

Rust, Eric C., Nature and Man in Biblical Thought, London (Lutterworth Press), 1953.

-, Science and Faith. Towards a Theological Understanding of $\mathrm{Na}$ ture, New York (Oxford University Press), 1967.

Schilling, Harold, Science and Religion; An Interpretation of Two Communities, New York (Charles Scribner's Sons), 1962. 
Shapley, Harlow, (Hg.), Science Ponders Religion, New York (Appleton-Century-Crofts, Inc.), 1960.

Sinnott, Edmund W., The Biology of the Spirit, New York (Viking Press), 1955.

-, Life and Mind, Yellow Springs, Ohio (Antioch Press), 1956.

-, Matter, Mind and Man. The Biology of Human Nature, New York (Harper \& Row), 1957.

-, Two Roads to Truth, New York (Viking), 1953.

Smethurst, Arthur F., Modern Science and Cbristian Beliefs, New York (Abingdon), 1955.

Snow, Charles P., The Two Cultures and the Scientific Revolution, New York (Cambridge University Press), 1961.

Sonneborn, Tracy M., (Hg.), The Control of Human Heredity and Evolution, New York (Macmillan), 19675.

Tax, Sol, (Hg.), Evolution after Darwin. The University of Chicago Centennial, 3 Bde., Chicago (University of Chicago Press), 1960.

Torrance, Thomas F., God and Rationality, London (Oxford University Press), 1971.

-, Theological Science. Based on the Hewett Lectures for 1959, London (Oxford University Press), 1969.

Vahanian, Gabriel, The Death of God. The Culture of Our PostChristian Era, New York (G. Braziller), 1961.

Van der Ziel, Aldert, Genesis and Scientific Inquiry, Minneapolis (T. S. Denison \& Co.), 1965.

-, The Natural Sciences and the Christian Message, Minneapolis (T. S. Denison \& Co.), 1960.

Waddington, Conrad H., The Nature of Life, London (George Allen \& Unwin Ltd.), 1961.

White, Andrew D., A History of the Warfare of Science with Theology in Christendom, New York (D. Appleton and Co.), 1910.

White, Edwand A., Science arod Religion in American Thought, New York (AMS Press), 1968.

White, Dale, (Hg.), Dialogue in Medicine and Theology, Nashville (Abingdon), 1967.

Whitehead, Alfred North, The Interpretation of Science. Selected Essays, hg. v. A. H. Johnson, Indianapolis (Bobbs-Merrill), 1961.

-, Process and Realty. An Essay in Cosmology, New York (Macmillan), $1960^{5}$.

-, Science and the Modern World. Lowell Lectures (1925), New York (Macmillian), 1960.

Yarnold, Greville D., The Spiritual Crisis of the Scientific Age, New York (Macmillan), 1959. 


\section{Zeitschriften}

Journal of the American Scienfitic Affiliation, Richard H. Bube (Hg.), Stanford University, Cal., 1941- (vierteljährlich).

Zygon, Journal of Religion and Science, Ralph Wendell Burhoe (Hg.), University of Chicago Press, 1966- (vierteljährlich).

Weitere Literaturangaben entnehme man den Anmerkungen. 